\title{
WILEY-VCH
}

DOI: $10.1002 /(($ please add manuscript number $))$

Article type: Full Paper

\section{Optical Acetone Vapor Sensors Based on Chiral Nematic Liquid Crystals and Reactive Chiral Dopants}

Pascal Cachelin, Joshua P. Green, Ton Peijs, Martin Heeney and Cees W. M. Bastiaansen*

P. Cachelin, Prof. C.W.M. Bastiaansen, Prof. T Peijs

Queen Mary University of London, Mile End Road, E1 4NS, United Kingdom

J. P. Green, Prof. M. Heeney

Imperial College London, Exhibition Road, SW7 2AZ, United Kingdom

Prof. $\quad$ C.W.M.

Technische Universiteit Eindhoven, Den Dolech 2, 5612, the Netherlands

Bastiaansen

C.W.M.Bastiaansen@tue.nl

Keywords: Chiral Nematic Liquid Crystals, Sensors, Acetone Vapor, Chemosensors

Accurate monitoring of exposure to organic vapors, such as acetone, is an important part of maintaining a safe working environment and adhering to long- and short-term exposure limits. Here, a novel acetone vapor detection system is described based on the use of a reactive chiral dopant in a nematic liquid crystal thin film, which can accurately monitor the total exposure over a given timeframe through changes in the reflection band of the film. It was found that films exposed to 1000 parts per million by volume (ppmv) of acetone vapor for two hours exhibited a shift in reflection band of $12 \mathrm{~nm}$, with smaller shifts observed at lower concentrations of acetone. Such sensors have potential applications in industrial hygiene.

\section{Introduction}

Recently, much attention has been paid towards the development of optical sensors, particularly for personal safety applications. ${ }^{[1]}$ These sensors are attractive as they can allow for analysis without requiring access to analytical facilities. Optical sensors based on liquid crystals (LCs) have been investigated extensively in the past. For instance, a broad range of optical sensors 


\section{WILEY-VCH}

was developed previously which are based on interfacial changes that influence the alignment of liquid crystals in response to analytes such as light, ${ }^{[2]}$ gasses, ${ }^{[3-6]}$ biomolecules $^{[7-9]}$ and aerosols ${ }^{[5]}$ which is covered in an excellent recent review by Carlton et al ${ }^{[10]}$ In these sensors, chemically functionalized self-assembled monolayers are employed which react with analytes that act as an alignment layer for liquid crystals. The change in birefringence is monitored upon exposure to an analyte via transmission measurements between crossed linear polarizers.

Optical sensors based on the chiral nematic phase were also explored in the past. Typically, these sensors operate on a change in the reflection band of the chiral nematic liquid crystal, which allows for optical monitoring without the need for polarizers. In most cases, these sensors operate by the absorption of the analyte causing swelling which in turn alters the cholesteric pitch. Such sensors have been previously described for alcohols, ${ }^{[11]}$ amines, ${ }^{[12,13]}$ VOCs $^{[14-16]}$ and gases like oxygen and carbon dioxide. ${ }^{[17]}$ These optical sensors can have a high sensitivity but are often also cross-sensitive to similar molecules. These sensors act as real-time sensors, and measure the level of analyte that is present. This is in contrast to time-integrating sensors, which can effectively act as a chemical dosimeters by measuring the total exposure to an analyte over a given time frame.

To date, only one time-integrating sensors based on chiral nematic liquid crystals has been reported. ${ }^{[18]}$ This sensor operated on the basis of a reactive chiral dopant in a nematic LC matrix that irreversibly reacted with water vapor, resulting in a change in the reflection band of the chiral nematic liquid crystals which was attributed to a change in the helical twisting power (HTP) of the dopant.

Detection of acetone vapor with a time-integrating sensor requires the use of a chiral dopant system capable of irreversibly reacting with acetone. TADDOLs (TetraAryl-1,3-Dioxolane4,5-DimethanOLs) are a group of compounds traditionally used in catalysis as chiral 


\section{WILEY-VCH}

auxiliaries. ${ }^{[19]}$ A secondary use is as chiral dopants in chiral-nematic liquid crystals, where they act as dopants with exceptionally high HTP. ${ }^{[20]}$ Previous work by Han et al ${ }^{[17]}$ has demonstrated that $R, R$-TADDOLs can be complexed with amines to produce a reactive dopant complex capable for the real-time detecting of $\mathrm{CO}_{2}$.

In this study, a new chiral nematic reactive dopant is reported and their use in timeintegrating sensors for acetone is explored. This dopant consists of R,R-TADDOL complexes with phenylhydrazine to generate reactive and irreversible systems with a high sensitivity to acetone and low cross sensitivity.

\section{Results and discussion}

2. Complex formation and testing

In this study, R,R-TADDOLs are again used as chiral auxiliaries. ${ }^{[18]}$ Phenylhydrazine (PH), which is capable of hydrogen-bonding to the TADDOL, is expected to have a high reactivity and selectivity towards ketones. The specific TADDOL-PH complex chosen is shown in Figure 1, and consists of $(4 R, 5 R)-2,2-$ Dimethyl- $\alpha, \alpha, \alpha^{\prime}, \alpha^{\prime}$-tetra(2-naphthyl)dioxolane-4,5-dimethanol complexed to $\mathrm{PH}$.

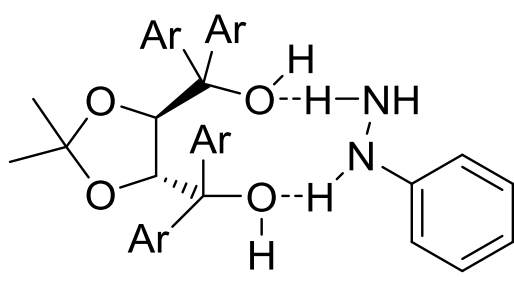

TADDOL-PH
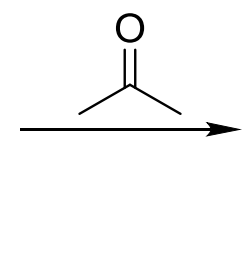

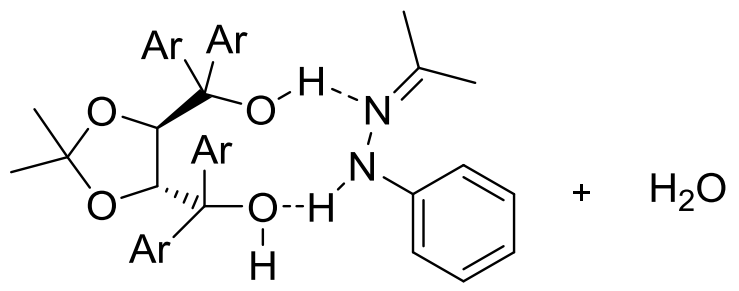

TADDOL-PAC<smiles>[Li]c1ccc2ccccc2c1</smiles>

Figure 1. The TADDOL-Phenylhydrazine (TADDOL-PH) complex, and the imine product of the reaction with acetone (TADDOL-PAc) 


\section{WILEY-VCH}

The formation of this complex was confirmed by ${ }^{1} \mathrm{H}$ NMR (Figure S1). The ${ }^{1} \mathrm{H}$ NMR spectrum of a 1:1 molar ratio mixture of the TADDOL-PH complex shows several important changes in comparison to those of samples of pure PH and TADDOL. The majority of the aromatic peaks in both TADDOL and PH are largely unaffected by complexation, with changes in chemical shifts of less than $0.02 \mathrm{ppm}$ seen for all peaks except those corresponding to the protons in ortho positions of phenylhydrazine, which shift upfield from around $6.83 \mathrm{ppm}$ to $6.74 \mathrm{ppm}$ in the presence of TADDOL. This is expected as the naphthyl protons on TADDOL are molecularly distant from the predicted complexation site, whilst the ortho position of PH is directly attached to the hydrazine functional group. The - $\mathrm{CH}$ protons on the TADDOL dioxolane ring show slight shielding from $4.97 \mathrm{ppm}$ to $4.94 \mathrm{ppm}$, whilst the methyl groups show no change from their original position. By far the largest shifts are seen for the protons corresponding to the functional groups (hydroxyl for TADDOL and hydrazinyl for PH). The protons of the latter shift upfield from 5.17 to $5.05 \mathrm{ppm}$ and 3.58 to $3.52 \mathrm{ppm}$ for the $-\mathrm{NH}$ - and $-\mathrm{NH} 2$ protons respectively, with the hydroxyl protons shifted downfield from $4.24 \mathrm{ppm}$ to $4.62 \mathrm{ppm}$. The fact that the hydrazinyl protons shift upfield and the hydroxyl protons downfield indicates that hydrogen bonds form between the two molecules with -NH/-NH2 acting as hydrogen bond donors and the $-\mathrm{OH}$ groups acting as hydrogen bond acceptors.

In order to determine the maximum shift in the reflection band resulting from full conversion of the hydrazine to imine product, it was first necessary to create the expected product of the reaction between TADDOL-PH and acetone (TADDOL-PAc, Figure 1). A reaction of PH with saturated acetone vapor was carried out. We found that the resultant product (PAc) was obtained quantitatively with and without the use of an acid catalyst (Figure S2). This was then combined with TADDOL to produce a TADDOL-PAc complex. 


\section{WILEY-VCH}

In order to determine the suitability of the TADDOL-PH system as a reactive dopant, reference experiments were performed to measure the HTP of the TADDOL complexes before and after reaction with acetone. LC/dopant mixtures were formulated and processed by spin-coating onto a rubbed polyimide alignment layer. The prepared samples yielded brightly colored films and optical microscopy revealed that the films exhibited a so-called fingerprint texture when viewed between crossed polarizers (Figure 2), indicating splay alignment at the LC-air interface. ${ }^{[21]}$ The films exhibit a reflection band at $493.5 \mathrm{~nm}$ for TADDOL, $499.5 \mathrm{~nm}$ for TADDOL-PH and $514.5 \mathrm{~nm}$ for TADDOL-Pac respectively. From this, the pitch and the HTP were calculated using enantiomeric excess $\left(e_{e}\right)$ and weight fraction $\left(c_{w}\right)$ of the dopant, as well as the average refractive index of the nematic liquid crystalline host $(\bar{n})$ (Equations 1, 2). This information is summarized in Table 1.

$$
p=\frac{\lambda}{\bar{n}}
$$

$$
H T P=\left(p e_{e} c_{w}\right)^{-1}
$$

The difference in the HTP values of TADDOL and TADDOL-PH provide a strong indication that the TADDOL-PH complex is formed, and the difference in the HTP values of TADDOL$\mathrm{PH}$ and TADDOL-PAc indicates the reaction with acetone produces the TADDOL-PAc compound. 


\section{WILEY-VCH}

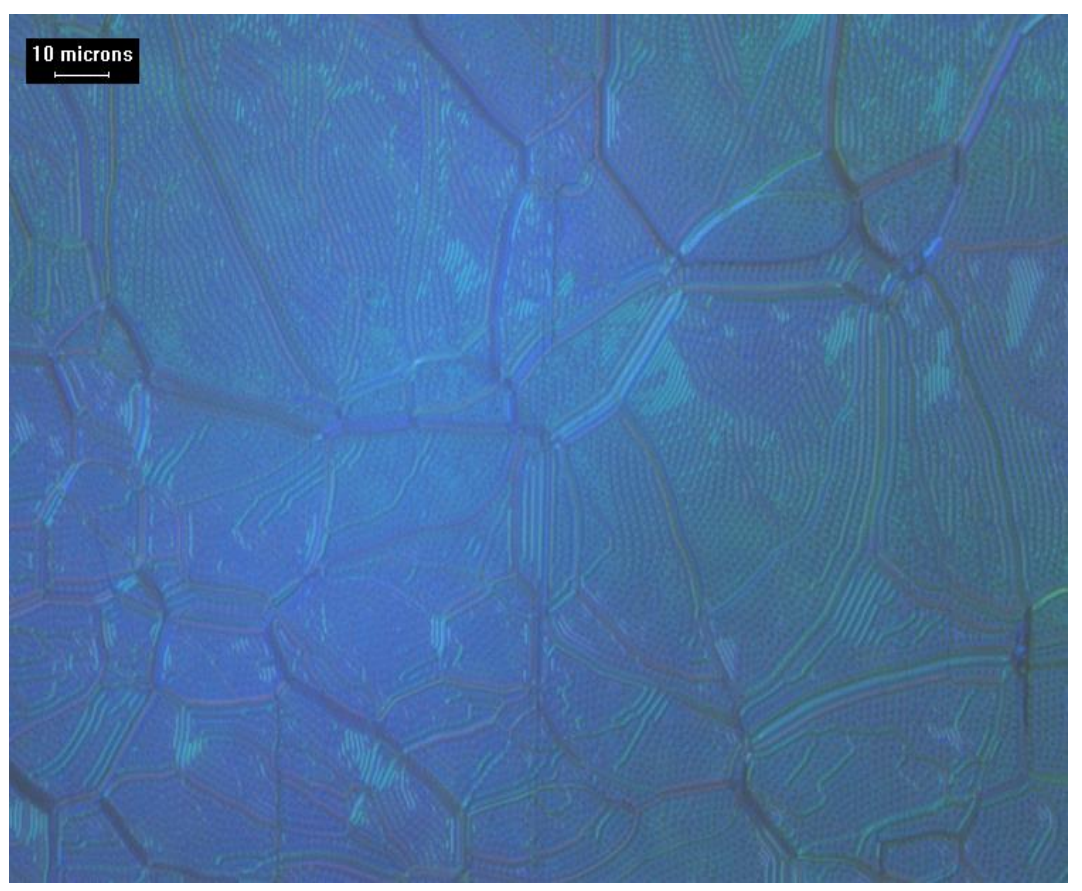

Figure 2. Optical Microscopy image between crossed polarizers showing the fingerprint texture. Scale bar $=10 \mu \mathrm{m}$

Table 1. Film compositions and resultant helical twisting powers.

\begin{tabular}{lllll}
\hline Dopant & $\begin{array}{l}\text { Concentration } \\
(\text { wt. \%) }\end{array}$ & $\begin{array}{l}\text { Reflection } \\
\text { maximum } \\
(\mathrm{nm})\end{array}$ & $\begin{array}{l}\text { Pitch } \\
(\mathrm{nm})\end{array}$ & $\begin{array}{c}\mathrm{HTP} \\
\left(\mu \mathrm{m}^{-1}\right)\end{array}$ \\
\hline & 3.44 & 493.5 & 324 & 90.62 \\
& & & & \\
\end{tabular}

As indicated in Equation 3 the pitch, and therefore the wavelength of the reflection band, is dependent on the doping concentration used. 


\section{WILEY-VCH}

In order to determine a suitable exposure time for this reaction, GCMS analysis of the bulk phase reaction of $\mathrm{PhNNH}_{2} / R, R$-TADDOL and 1000 p.p.m.v. acetone vapor in E7 solvent was carried out. It was found that the reaction proceeded to $60 \%$ conversion over 120 minutes, after which it plateaued (Figure S3). Extended exposure ( $>24$ hours) did not lead to any increase in conversion. The time frame of 2 hours was therefore used as the basis for subsequent experiments.

\subsection{Sensitivity to acetone vapor}

To test the sensitivity of the sensor, chiral nematic films were prepared containing $4 \%$ of chiral dopant. It was reported previously that exposure to saturated acetone results in complete dissolution of the $\mathrm{LC}^{[22]}$ and therefore this study is limited to low acetone levels such as encountered in industrial hygiene.

CNLC films manufactured with the TADDOL-PH showed an irreversible red-shift of $12 \mathrm{~nm}$ in absorption maximum compared to before acetone exposure (Figure 3c), while those of control films made with TADDOL-PAc and pure TADDOL showed no change in absorption (Figure 3a,b) compared to before acetone exposure. This indicates that the change observed for the films made with TADDOL-PH is due to a reaction between the dopant and the analyte, and not due to a swelling of the nematic host. As shown in Table 1, full conversion of PH to PAc would give a red-shift in absorption maximum of $14 \mathrm{~nm}$, with the observed $12 \mathrm{~nm}$ shift equating to an $85 \%$ conversion. 


\section{WILEY-VCH}
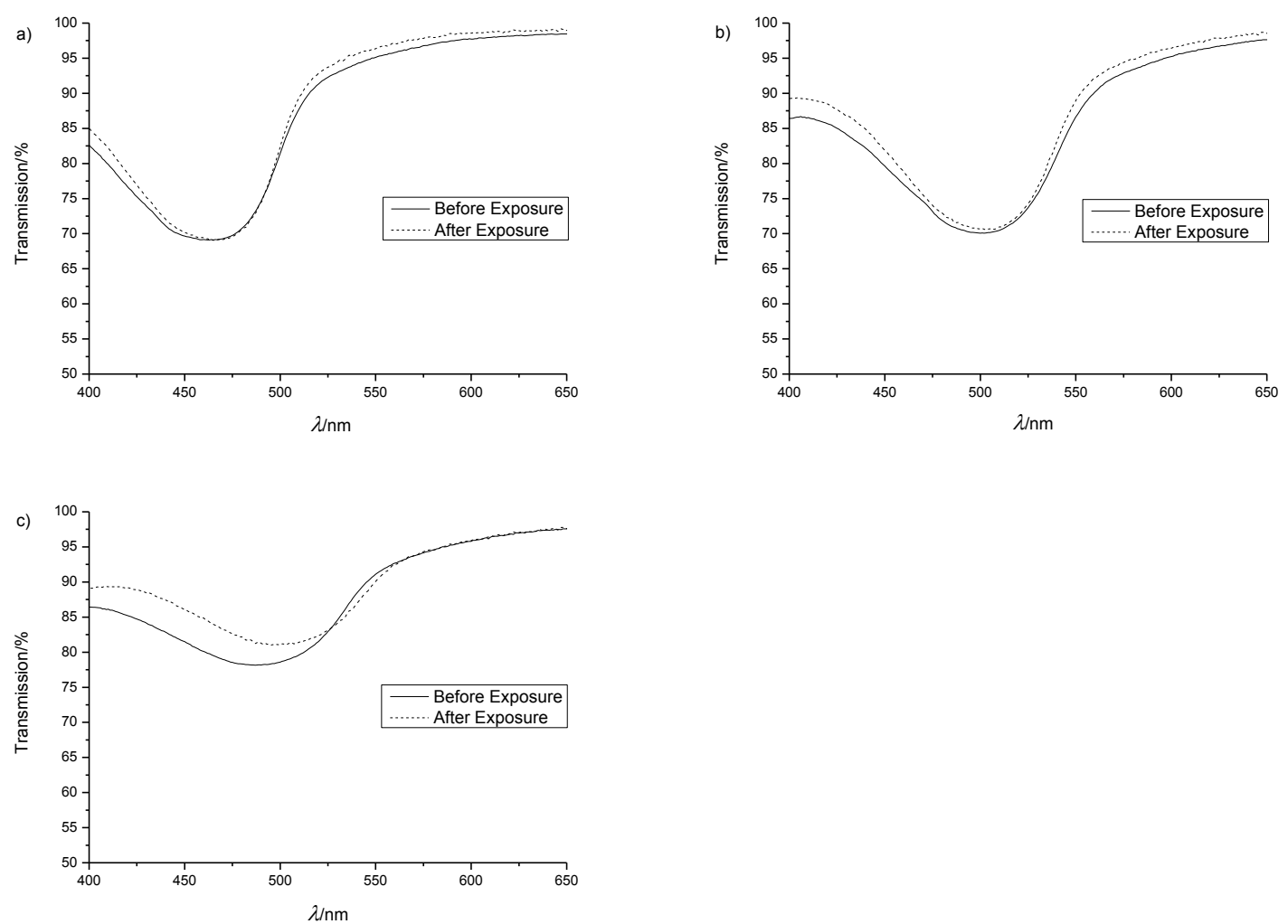

Figure 3. The UV/Vis absorption spectra of a) TADDOL, b) TADDOL-PAc and c) TADDOL$\mathrm{PH}$ before and after exposure to acetone vapor.

A series of experiments consisting of exposing CNLC films to a variety of acetone concentrations was carried out. It was found that the operational range of the detector is in the $100-1000$ ppmv range of acetone vapor over a time frame of two hours with the absorption maximum exhibiting a close to linear relationship in with acetone concentration up to approximately 1000 ppmv (Figure 4). 


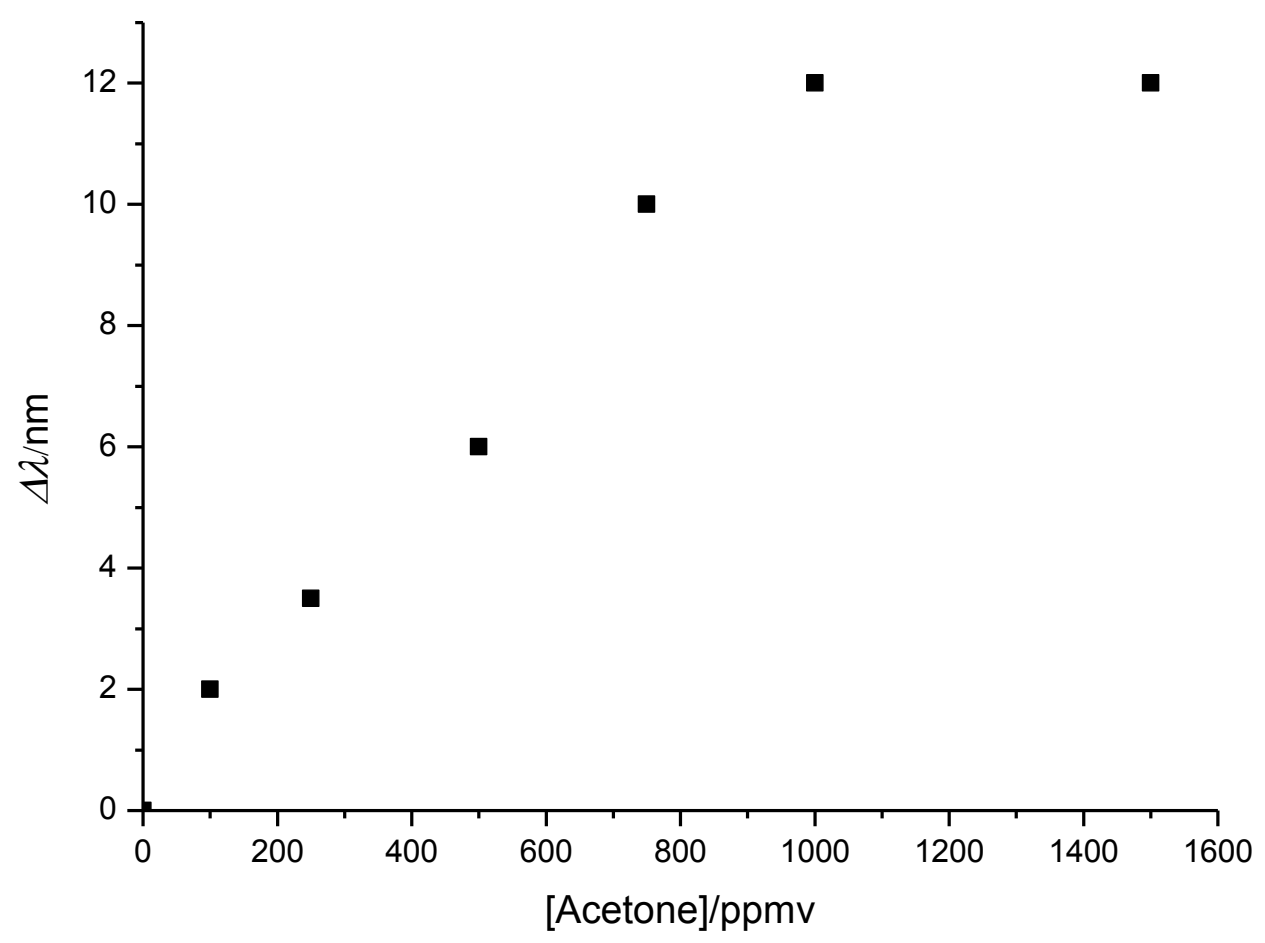

Figure 4. Change in absorption maximum $(\Delta \lambda)$ of CNLC sensors exposed acetone vapor.

As shown in Figure 4, concentrations above 1,000 ppmv did not result in any change in the reflection band of the sample, indicating saturation of the sensor. Similarly, concentrations below 100 ppmv did not produce a measurable shift. The sensitivity of the sensor in the intermediate region was calculated from the slope and was $0.0012 \mathrm{~nm} / \mathrm{ppmv}$.

\subsection{Cross-sensitivity}

A key requirement for any sensor is a low cross-sensitivity to other components of a potential analyte mixture. In order to quantify this cross-sensitivity, experiments were performed to test the response of the sensor to an atmosphere of 10,000 ppmv of other industrial chemicals, such as isopropyl alcohol, ethanol, and water vapor, for a 24 hours. It was found that there was no change in absorption maximum after exposure to ethanol or water, although a $4 \mathrm{~nm}$ redshift was seen after exposure to isopropyl alcohol vapor. 


\section{WILEY-VCH}

\section{Discussion}

Acetone is a widely used industrial solvent, with many varied applications in different industries. Although relatively harmless at low concentrations for short times, chronic exposure to acetone vapor can lead to a progression of symptoms from irritation of mucous membranes to headaches, nausea, loss of coordination, central nervous system depression and finally, at sufficiently high exposure levels, cardiorespiratory failure. ${ }^{[23-27]}$ Exposure to levels as low as 250 ppmv for 4 hours has been shown to lead to neurobehavioral effects, including increased aggression in males. ${ }^{[28]}$ As such, monitoring exposure to acetone vapor and ensuring that it falls within safety guidelines is an important aspect of industrial hygiene.

The industrial guidance issued by the American Conference of Governmental Industrial Hygienists on acetone vapor gives a maximum time weighted average (TWA) of $750 \mathrm{ppmv}$ and a short term exposure limit (STEL) of 1000 ppmv, while the Workplace Exposure Limit set by the UK Health and Safety Executive is 500 ppmv for the TWA and 1500 ppmv for the STEL. The sensor developed in this work is capable of monitoring the TWA for both these standards over a time frame of two hours, although maximum change in the reflection band of $12 \mathrm{~nm}$ is too small to be monitored visually, and currently requires spectrometric analysis.

Another potential application for acetone sensors is monitoring blood-glucose levels in the case of type 1 diabetes by using breath acetone level as a proxy, ${ }^{[29,30]}$ although significant debate remains over the validity of breath acetone as a suitable proxy. ${ }^{[31,32]}$ If acetone is shown to be a reliable biomarker then acetone sensors have the potential for diagnostic and monitoring applications in diabetes management. Such a sensor would need to be able to detect very low concentrations of acetone vapor, typically in the range of $0.1-10 \mathrm{ppmv}$. This is significantly below the capability of the sensors developed here. 


\section{WILEY-VCH}

4. Conclusions

A chiral nematic sensor for acetone was developed that exhibits a low sensitivity $(0.0012$ $\mathrm{nm} / \mathrm{ppmv}$ ) and a low cross-sensitivity. The sensor was based on a TADDOL hydrazine complex as a reactive chiral dopant within a nematic LC that irreversibly reacts with acetone, resulting in a change in the reflection band of the sensor. It is shown that this sensor is potentially useful for industrial hygiene applicants and not for the detection of diabetes via breath analysis which is related to the high sensitivity that is needed.

\section{Experimental Section}

Materials and sample preparation: All materials were used as received. Glass slides (Fischer Scientific) were washed with isopropyl alcohol and $\mathrm{N}_{2}$ dried prior to spin coating with polyimide precursor AL1051 (JSR Micro). The substrates were thermally cured $\left(200{ }^{\circ} \mathrm{C}\right.$ for two hours). The coated glass slides were then uniaxially rubbed with a velvet cloth in order to induce a planar alignment layer.

The PAc imine product was formed by exposing phenylhydrazine (Alfa Aesar) to a saturated acetone vapor under argon for 24 hours. The PAc was obtained quantitatively and used without further purification.

CNLC mixtures were formed by dissolving $R, R$-TADDOL (Sigma-Aldrich) and phenylhydrazine in E7 (Synthon Chemicals). The resultant mixture was diluted 1:1 w/w with p-xylene (Sigma-Aldrich) in order to decrease the viscosity ahead of spin-coating.

GCMS experiment: $\mathrm{PhNNH}_{2}$ was dissolved in $\mathrm{E} 7$ and exposed to 1000 p.p.m.v. acetone in an argon atmosphere for the lengths of time shown in the graph. Samples were then removed from 


\section{WILEY-VCH}

the reaction vessel, dissolved in dichloromethane and submitted for GCMS analysis. Peaks corresponding to $\mathrm{PhNNH}_{2}$ and $\mathrm{PhNNAc}$ (as determined by the presence of ions at $\mathrm{m} / \mathrm{z}=108$ and $\mathrm{m} / \mathrm{z}=148$ respectively) were integrated using Origin Pro software, and the relative areas plotted as a function of reaction time.

Formation of thin films by spin-coating: The CLC mixtures were spin-coated onto the coated glass slides. After spin-coating the samples were briefly heated to just above the clearing point of the mixture $\left(65^{\circ} \mathrm{C}\right)$ in order to aid evaporation of any residual xylene solvent.

Solvent vapor exposure testing: Samples were placed inside a 2 L 3-necked-flask and the necks were sealed with Suba-Seal septa. The flask was flushed with nitrogen gas to remove any residual solvent vapor, and then acetone, ethanol, isopropyl alcohol (Sigma-Aldrich) or deionized water was injected through the septa using a microsyringe. Unless otherwise stated, samples were exposed for 2 hours before characterization, after which they were briefly heated above the isotropic point in order to remove any residual solvent.

Characterization: UV spectra were taken using a Lambda 950 UV spectrometer (Perkin Elmer) using non-polarized light. GCMS data were obtained using Agilent Technologies 7890A gas chromatograph connected to an Agilent 5975C detector. ${ }^{1} \mathrm{H}$ and ${ }^{13} \mathrm{C}$ NMR spectra were recorded using Bruker AV-400 spectrometers $\left({ }^{1} \mathrm{H}=400 \mathrm{MHz},{ }^{13} \mathrm{C}=101 \mathrm{MHz}\right)$ at $298 \mathrm{~K}$.

\section{Supporting Information}

Supporting Information is available from the Wiley Online Library or from the author.

\section{Acknowledgements}

We thank the UK's Engineering and Physical Sciences Research Council (EPSRC) for financial support via the Doctoral Training Centre in Plastic Electronics EP/G037515/1. We gratefully acknowledge Dr. Olivier Picot for advice and helpful discussions. 


\section{WILEY-VCH}

Received: ((will be filled in by the editorial staff))

Revised: ((will be filled in by the editorial staff))

Published online: ((will be filled in by the editorial staff))

[1] A. Lobnik, Intelligent Textiles for Personal Protection and Safety, IOS Press, Amsterdam, 2006.

[2] H. Knobloch, H. Orendi, M. Buchel, T. Seki, S. Ito, W. Knoll, J. Appl. Phys. 1995, 77, 481.

[3] J. L. Adgate, A. Barteková, P. C. Raynor, J. G. Griggs, A. D. Ryan, B. R. Acharya, C. J. Volkmann, D. D. Most, S. Lai, M. D. Bonds, J. Environ. Monit. 2009, 11, 49.

[4] R. Nandi, S. K. Singh, H. K. Singh, B. Singh, R. K. Singh, Chem. Phys. Lett. 2014, 614, 62.

[5] K. D. Cadwell, N. a. Lockwood, B. a. Nellis, M. E. Alf, C. R. Willis, N. L. Abbott, Sensors Actuators, B Chem. 2007, 128, 91.

[6] R. R. Shah, N. L. Abbott, Langmuir 2003, 19, 275.

[7] S. R. Kim, N. L. Abbott, Adv. Mater. 2001, 13, 1445.

[8] S. Zhong, C.-H. Jang, Biosens. Bioelectron. 2014, 59, 293.

[9] H. Tan, S. Yang, G. Shen, R. Yu, Z. Wu, Angew. Chemie - Int. Ed. 2010, 49, 8608.

[10] R. J. Carlton, J. T. Hunter, D. S. Miller, R. Abbasi, P. C. Mushenheim, L. N. Tan, N. L. Abbott, Liq. Cryst. Rev. 2013, 1, 29.

[11] C.-K. Chang, C. W. M. Bastiaansen, D. J. Broer, H.-L. Kuo, Macromolecules 2012, 45, 4550 .

[12] N. Kirchner, L. Zedler, T. G. Mayerhöfer, G. J. Mohr, Chem. Commun. (Camb). 2006, 1512.

[13] L. Sutarlie, H. Qin, K.-L. Yang, Analyst 2010, 135, 1691.

[14] D. A. Winterbottom, R. Narayanaswamy, I. M. Raimundo, Sensors Actuators, B Chem. 2003, 90, 52 .

[15] F. Dickert, A. Haunschild, P. Hofmann, Fresenius. J. Anal. Chem. 1994, 577.

[16] A. Mujahid, H. Stathopulos, P. A. Lieberzeit, F. L. Dickert, Sensors 2010, 10, 4887.

[17] Y. Han, K. Pacheco, C. W. M. Bastiaansen, D. J. Broer, R. P. Sijbesma, J. Am. Chem. Soc. 2010, 132, 2961. 


\section{WILEY-VCH}

[18] A. Saha, Y. Tanaka, Y. Han, C. M. W. Bastiaansen, D. J. Broer, R. P. Sijbesma, Chem. Commun. (Camb). 2012, 48, 4579.

[19] D. Seebach, A. K. Beck, A. Heckel, Angew. Chem. Int. Ed. Engl. 2001, 40, 92.

[20] R. Eelkema, B. L. Feringa, Org. Biomol. Chem. 2006, 4, 3729.

[21] P. J. Collings, Liquid Crystals, Adam Hilger, Bristol, 1990.

[22] C. K. Chang, S. W. Chiu, H. L. Kuo, K. T. Tang, Appl. Phys. Lett. 2012, 100, 2013.

[23] J. H. E. Arts, J. Mojet, L. J. van Gemert, H. H. Emmen, J. H. C. M. Lammers, J. Marquart, R. a Woutersen, V. J. Feron, Crit. Rev. Toxicol. 2002, 32, 43.

[24] G. E. Ziem, B. I. Castleman, J. Occup. Med. 1989, 31, 910.

[25] A. Fujino, T. Satoh, T. Takebayashi, H. Nakashima, H. Sakurai, T. Higashi, H. Matumura, H. Minaguchi, T. Kawai, Br. J. Ind. Med. 1992, 49, 654.

[26] E. Wigaeus, S. Holm, I. Åstrand, Scand. J. Work. Environ. Health 1981, 7, 84.

[27] S. Kumagai, I. Matsunaga, T. Tabuchi, Am. Ind. Hyg. Assoc. J. 1998, 59, 242.

[28] R. B. Dick, J. V Setzer, B. J. Taylor, R. Shukla, Br. J. Ind. Med. 1989, 46, 111.

[29] A. Manolis, Clin. Chem. 1983, 29, 5.

[30] K.-H. Kim, S. A. Jahan, E. Kabir, TrAC Trends Anal. Chem. 2012, 33, 1.

[31] P. Spaněl, K. Dryahina, A. Rejšková, T. W. E. Chippendale, D. Smith, Physiol. Meas. 2011, 32, N23.

[32] P. Spaněl, D. Smith, Curr. Opin. Clin. Nutr. Metab. Care 2011, 14, 455.

A novel sensor based on a chiral nematic liquid crystal doped with a reactive dopant is

fabricated and investigated. It is found that the sensor acts as a real-time integrator, responding to total acetone dose by changing the wavelength of the reflection maximum of the chiral nematic liquid crystal. The sensitivity and cross sensitivity of the sensor are investigated.

\section{Keyword: Chiral Nematic Liquid Crystals, Sensors, Acetone Vapor, Chemosensors}




\section{WILEY-VCH}

Pascal Cachelin, Joshua P. Green, Ton Peijs, Martin Heeney and Cees W. M. Bastiaansen*

Optical Acetone Vapor Sensors Based on Chiral Nematic Liquid Crystals and Reactive Chiral Dopants

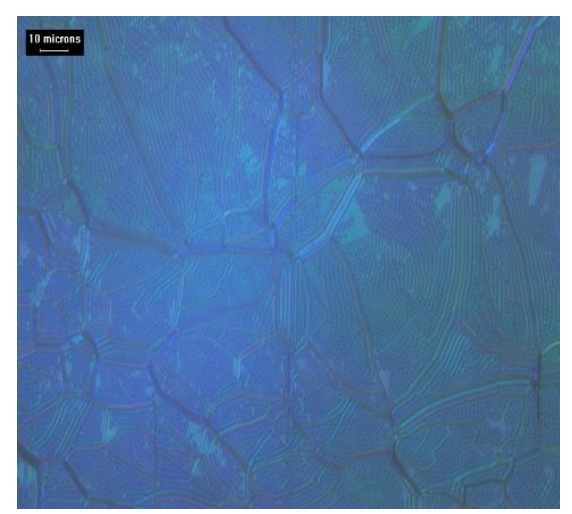

Copyright WILEY-VCH Verlag GmbH \& Co. KGaA, 69469 Weinheim, Germany, 2013.

Supporting Information

Optical Acetone Vapor Sensors Based on Chiral Nematic Liquid Crystals Thin Films Using Reactive Chiral Dopants

Pascal Cachelin, Joshua Green, Dr. Martin Heeney and Prof. Cees Bastiaansen

Figure S1. ${ }^{1} \mathrm{H}$ NMR spectra of TADDOL, PH, and the TADDOL-PH complex 


\section{WILEY-VCH}

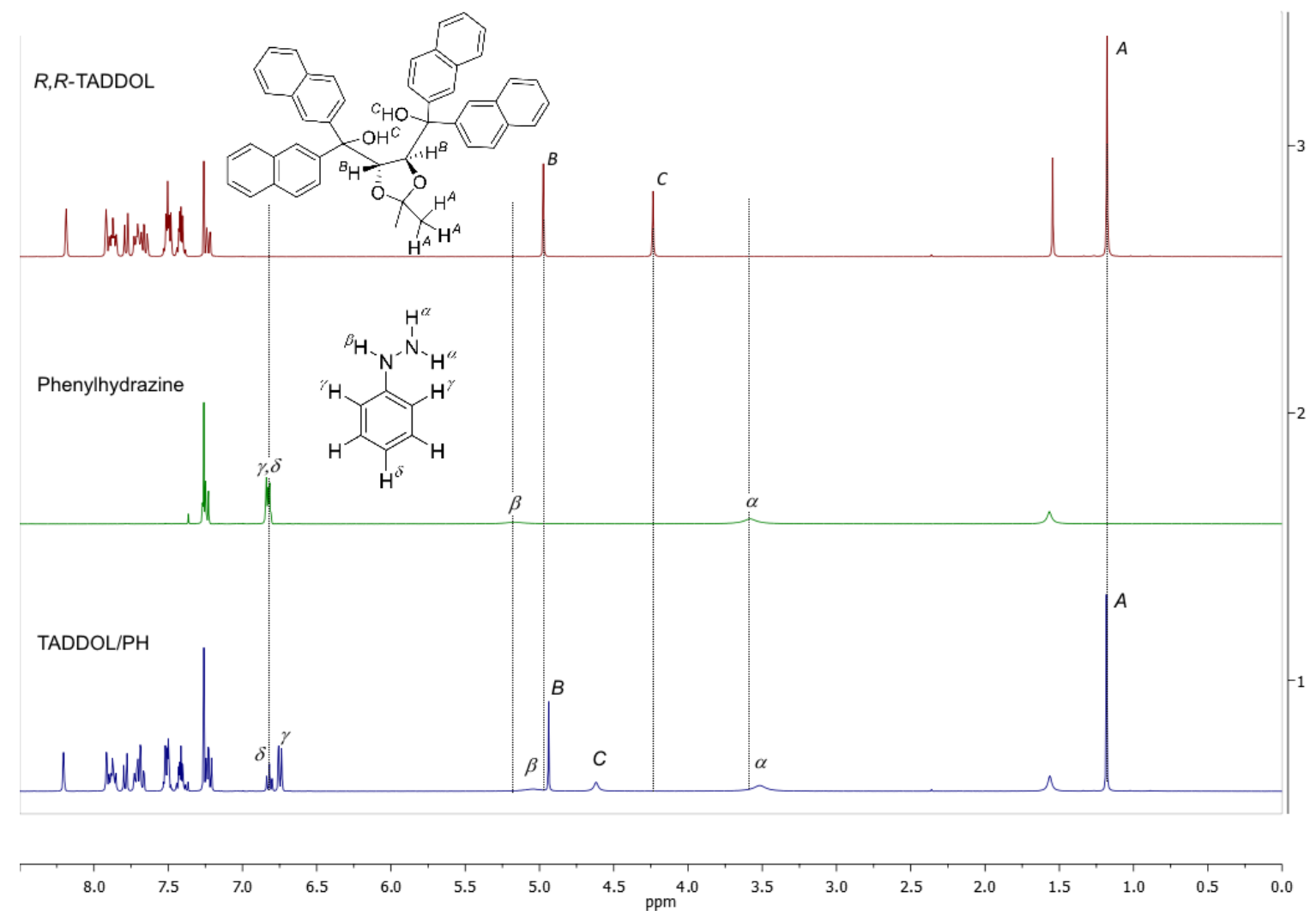

Spectra are ${ }^{1} \mathrm{H}$ NMR of TADDOL, $\mathrm{PH}$, and complex of the two at 1:1 molar equivalents, displayed in order from top to bottom. All spectra taken in $\mathrm{CDCl}_{3}$. Peaks relevant to the discussion are highlighted with dotted lines. The peaks at $7.26 \mathrm{ppm}$ and $1.56 \mathrm{ppm}$ are assigned to residual solvent $\left(\mathrm{CDCl}_{3}\right)$ and water respectively.

Figure S2. ${ }^{1} \mathrm{H}$ NMR of PAc 
WILEY-VCH

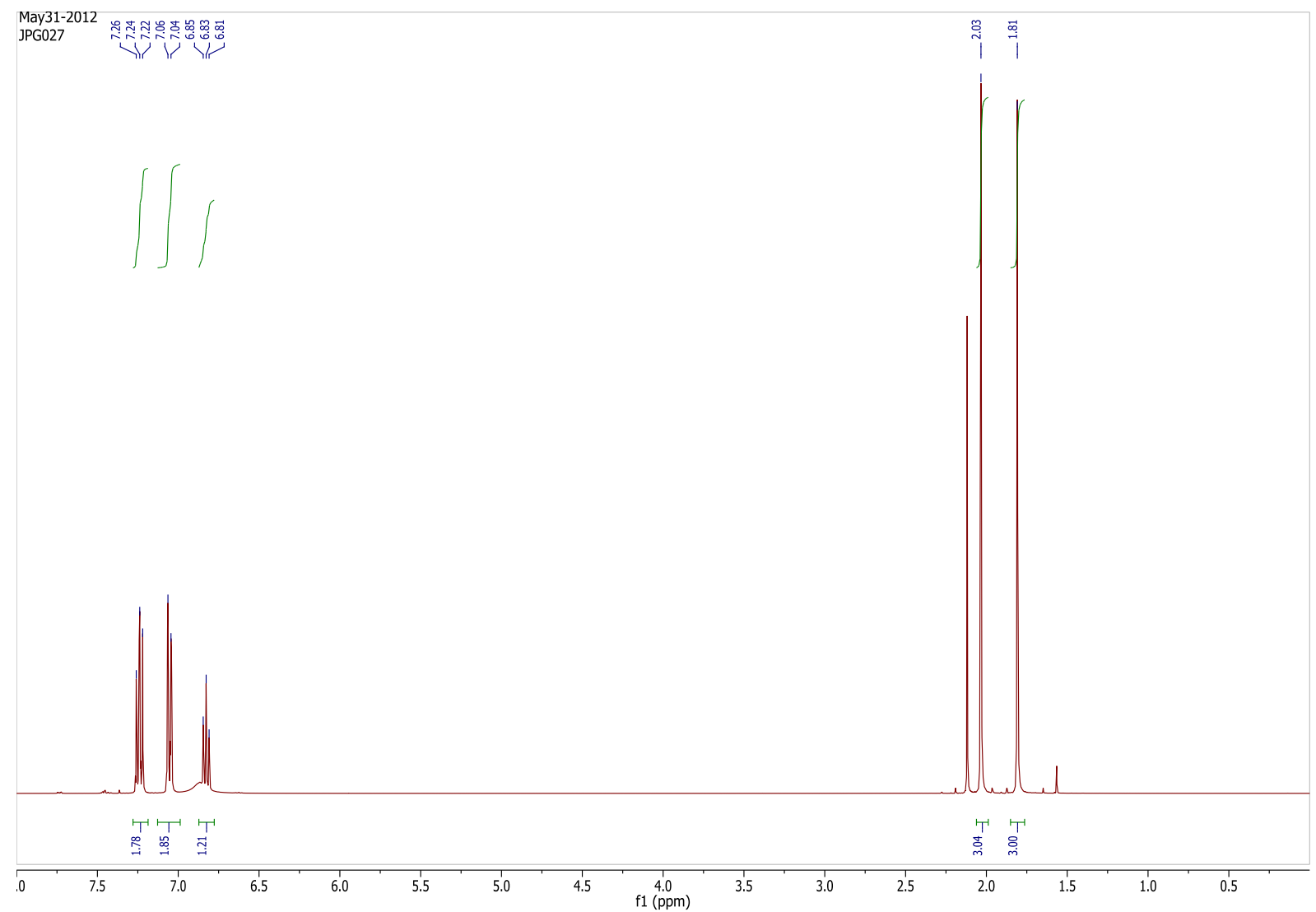

${ }^{1} \mathrm{H}$ NMR of PAc. The sample was prepared by exposing PH to saturated acetone vapor under argon for 24 hours, with no additional purification steps. The unassigned peak corresponds to unreacted acetone.

Figure S3. GCMS analysis of the reaction between PH and acetone. 
WILEY-VCH

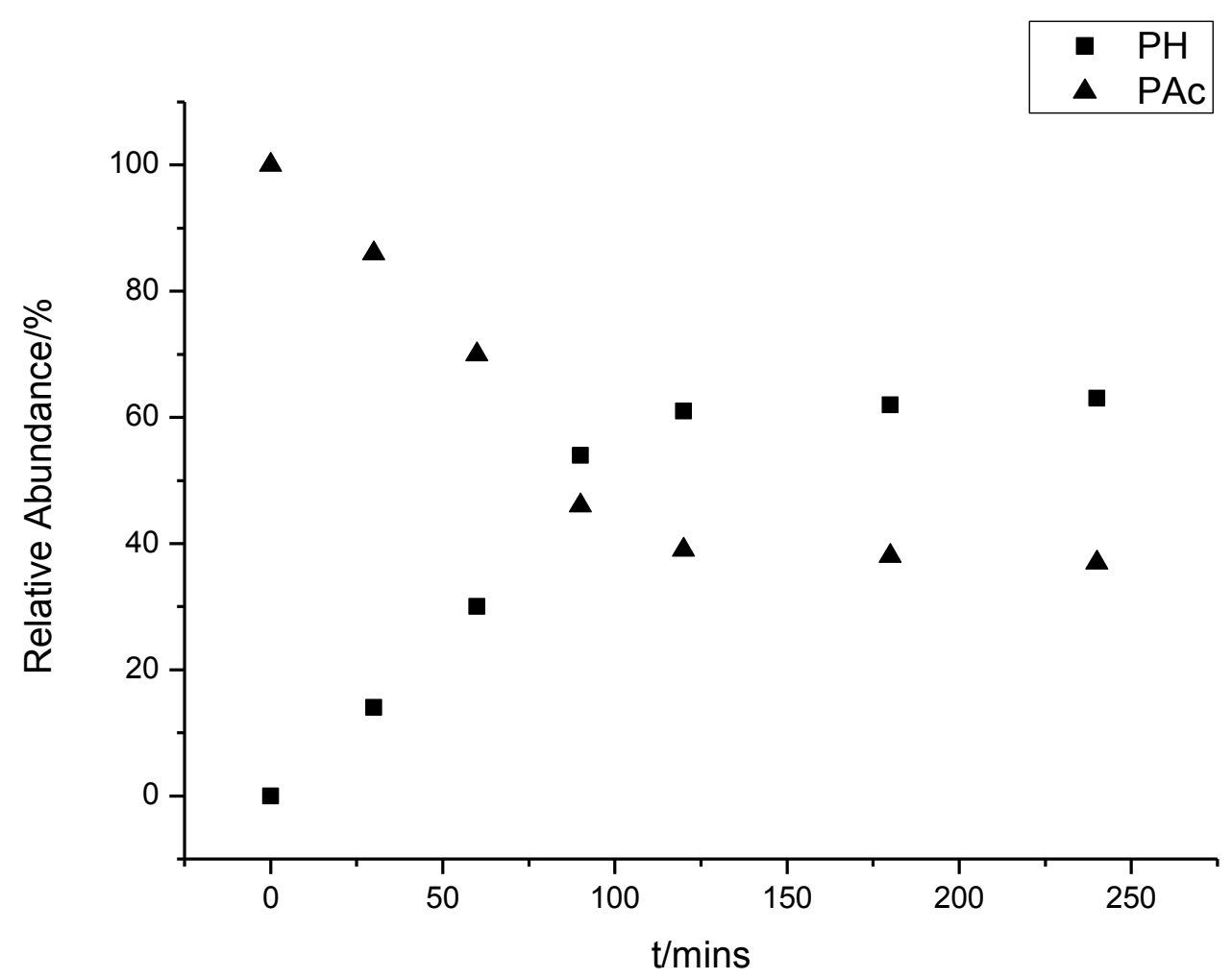

Figure S3 depicts the change in relative amounts of $\mathrm{PhNNH}_{2}$ and $\mathrm{PhNNAc}$ over the course of four hours as measured by GCMS. 